\title{
Assessment of Functionality and Diagnosis Precision of Digital Breast Tomosynthesis in Comparison with Breast Ultrasound Among Women in Risk of Breast Cancer
}

\author{
Ali Ghafari, ${ }^{1, *}$ and Ammar Nabiloo ${ }^{2}$ \\ ${ }^{1}$ Tabriz Paramedical School, Tabriz, Iran \\ ${ }^{2}$ Tabriz University of Medical Sciences, Tabriz, Iran \\ "Corresponding author: Ali Ghafari, Tabriz Paramedical School, Tabriz, Iran. E-mail: Aligh1101291@gmail.com
}

Received 2016 December 21; Accepted 2017 February 08.

\begin{abstract}
Background: Tomosynthesis is a new technology of digital mammography that enables the acquisition of a three - dimensional image and allows visualization of cancers not apparent by conventional mammography. Ultrasound (US) is also used for tumor monitoring, which is highly operator dependent and has some problems with reproducibility. US is also an easily available and noninvasive modality. Our goal is to have a comparison between breast ultrasound and tomosynthesis to determine which modality is better.

Methods: In a prospective study of 200 consecutive women using the DBT and MRI added to digital mammography (DM) and ultrasound (US) imaging techniques. Accuracy measurements were estimated using a lesion-by-lesion analysis for unifocal, multifocal/multicentric, bilateral and all carcinomas. They also calculated sensitivity according to breast density.

Results: DBT had a higher sensitivity than digital mammography alone and also had higher sensitivity in non-dense breast compared to dense breasts. Ultrasound had higher sensitivity in dense breasts. Ultrasound may result in false-negative findings, especially in the absence of a discrete mass. Digital breast tomosynthesis enables the production of images that eliminate the problem of superimposition of breast tissue, particularly in women with dense breast parenchyma.

Conclusions: Ultrasound had better detection functionality in dense breasts while it was unable to discriminate between lesion types due to tissue overlap requiring biopsy for further examinations. DBT is designed for the purpose of resolving tissue overlap especially when ultrasound modality was unable to determine lesion types. Further examinations and studies should be hold to determine which modality has better overall economical and detection efficiency.
\end{abstract}

Keywords: Tomosynthesis, Ultrasound, Breast Cancer, Mammography

This is an abstract presented in the 33rd Iranian congress of radiology (ICR) and the 15th congress of Iranian radiographic science association (IRSA) 